\title{
LANDMARKS IN CHEMOTHERAPY
}

$\mathrm{T}$ HE forty-ninth Bedson Lecture was delivered by Prof. C. H. Browning at King's College, Newcastle-upon-Tyne, on March 11, the subject being "Landmarks in Chemotherapy". After defining chemotherapy and summarizing its position in 1900 , Prof. Browning traced the search for synthetic compounds with curative action in infections due to trypanosomes, spirochaetes, malarial parasites and bacteria. The first great advance was the discovery of trypan red, with which Ehrlich and Shiga (1904) cured a surely fatal infection due to certain trypanosomes in mice. It had the disadvantage of staining the body tissues and was of little practical value; but it led to Bayer-205, a colourless symmetrically substituted urea derivative. In the case of the latter the ratio of the maximum tolerated to the minimum curative dose is very large; any change, however slight, in its molecular structure causes a great diminution in its activity. It is the most effective drug for the cure of the African forms of human trypanosomiasis, provided that treatment is begun early. About the same time as the discovery of trypan red, the organic arsenical atoxyl was shown by Thomas at Liverpool to be active against trypanosomes in vivo. Although inactive in vitro, it becomes active in the test tube on reduction to the trivalent arsenic state. Therefore it seems that the drug is reduced in the body or even inside the trypanosome itself. The outcome of the investigation of arsenicals in Ehrlich's laboratory was salvarsan and its derivative neosalvarsan. The latter, especially from convenience in use, has come to be the most important drug in the treatment of syphilis.

For the treatment of malaria, in addition to quinine, Ehrlich and Guttmann in 1891 found methylene blue to be active in the quartan variety. In 1926 Roehl showed that a quinoline derivative, plasmoquine, was effective; it destroyed the sexual stage or crescent of the tropical variety, which is resistant to other drugs. Schulemann (1932) introduced atebrin, an acridine derivative which, like quinine, acts on the asexual stage. It is noteworthy that both these synthetic anti-malarial drugs possess the same sidechain--NH.CH $\left(\mathrm{CH}_{3}\right) \cdot\left(\mathrm{CH}_{2}\right)_{3} \cdot \mathrm{N}\left(\mathrm{C}_{2} \mathrm{H}_{5}\right)_{2}$-although they differ in the stage of the parasite which is attacked.

Scientific attempts to destroy bacteria in the tissues date from 1867, when Lister experimented with some success on the use of crude carbolic acid in the treatment of compound fractures. This was an example of the application of a 'surface' antiseptic which could be brought into intimate contact with an in. fected site.

Up to about 1914 it was believed that an antiseptic must be a general protoplasm poison, harmful alike to the bacteria and the infected host. But this idea was disproved by observations with the newer antiseptics, such as acriflavine and proflavine. An example is afforded by Blake's experiments on trout ova infected with $B$. salmonicida; although carbolic acid is almost equally toxic to both, a concentration of 1 : 2,000 acriflavine will kill the bacteria without appreciably damaging the ova. Experimental streptococcal infections have also been successfully treated with acriflavine as well as certain quino. line compounds of the Schiff's base type (anils), the method being to inject the organisms into the abdominal cavity and then after an interval to follow up with a solution of the drug. Such antiseptics are valuable for the treatment of local infections of wounds, especially as preventives.

As regards the treatment of general bacterial infections, in 1911 Morgenroth and Levy showed ethylhydrocupreine (optoquine) to be active against experimental pneumococcal infection in mice; this observation was of scientific interest only. The first compounds of practical value were the azo dyes derived from $p$-aminobenzene sulphonamide, used by Domagk, such as "prontosil soluble". Tréfouel et al. showed that it is the sulphonamide part of the molecule which is effective, and that the removal of the part linked by the azo grouping does not cause loss of activity. Sulphanilamide itself is fairly soluble and rapidly absorbed and excreted. Analogues, such as sulphapyridine, which have proved very useful clinically, are much less soluble and more slowly absorbed, so tending to maintain a more continuous action. Compounds of this class which are effective against streptococci in experimental infections, may fail with staphylococci.

While these drugs have revolutionized the treatment of meningococcal meningitis and gonococcal infections, and have profoundly modified the course of pneumonia, they appear to have little effect on some other infections, for example, tuberculosis.

\section{CONTROL OF TRANSPORT IN GREAT BRITAIN}

$\mathrm{W}$ HILE Lord Stamp and his fellow chairmen of the four great railway companies in Great Britain have made spirited replies to some of the criticism levelled at the railways in recent months, rail transport is only one aspect of a problem which has aroused widespread concern. The importance of transport in war has been well brought home to us in recent months by the measures designed to save shipping space, and the severity of the attacks on British and Allied shipping. It is only in a limited sense, however, that we have learnt that the power to transport may be the decisive factor, although its bearing on our blockade of Germany has been indicated by Dr. Hugh Dalton. That blockade can only be decisive if transport difficulties, intensified by bombing, reduce the enemy's supplies in his vast territories below what Dr. Dalton calls the 'distributional minimum'. We have yet to appreciate as a nation that the transport problem is a simple problem, and must be governed by national, not by commercial, considerations. The transport services in Great Britain must make their utmost contribu. tion to the war effort without regard to other considerations. 